\title{
Simulation Study on the Balance of Glycolytic ATP Production and Oxidative Phosphorylation in Embryonic and Adult Ventricular Cells
}

\author{
Hitomi I Sano ${ }^{1,2}$, Yasuhiro Naito ${ }^{1,2,3}$, Masaru Tomita ${ }^{1,2,3}$ \\ ${ }^{1}$ Institute for Advanced Biosciences, Keio University, Kanagawa, Japan \\ ${ }^{2}$ Department of Environment and Information Studies, Keio University, Kanagawa, Japan \\ ${ }^{3}$ Systems Biology Program, Graduate School of Media and Governance, Keio University, \\ Kanagawa, Japan
}

\begin{abstract}
Here, we integrated various characteristics of fetal ventricular cells based on a mathematical model and predicted the contribution of each characteristic to maintenance of intracellular ATP concentration and sarcomere contraction under anoxic conditions. Our simulation results showed that higher glycogen content, higher hexokinase activity, and lower creatine concentration helped to prolong the time that contraction of ventricular cells was maintained under anoxic conditions. The integrated model enabled us to quantitatively assess the contributions of factors related to energy metabolism in ventricular cells.
\end{abstract}

\section{Introduction}

The developmental program of the heart requires accurate regulation to ensure continuous circulation and simultaneous cardiac morphogenesis, because any functional abnormalities may progress to congenital heart malformation. Energy metabolism in fetal ventricular cells is regulated differently from that in adult ventricular cells: fetal cardiomyocytes generally have immature mitochondria, and fetal ventricular cells show greater dependence on glycolytic ATP production (Figure 1). Because fetal cardiomyocytes show similar energy metabolic profiles to stem cell-derived cardiomyocytes and cardiomyocytes in the failing heart, an improved understanding of fetal cardiomyocytes will contribute to understanding processes in stem cell-derived cardiomyocytes and cardiomyocytes under pathological conditions.

In the present study, we demonstrated that greater glycogen content, higher hexokinase activity, and lower levels of total creatine all help to prolong the maintenance

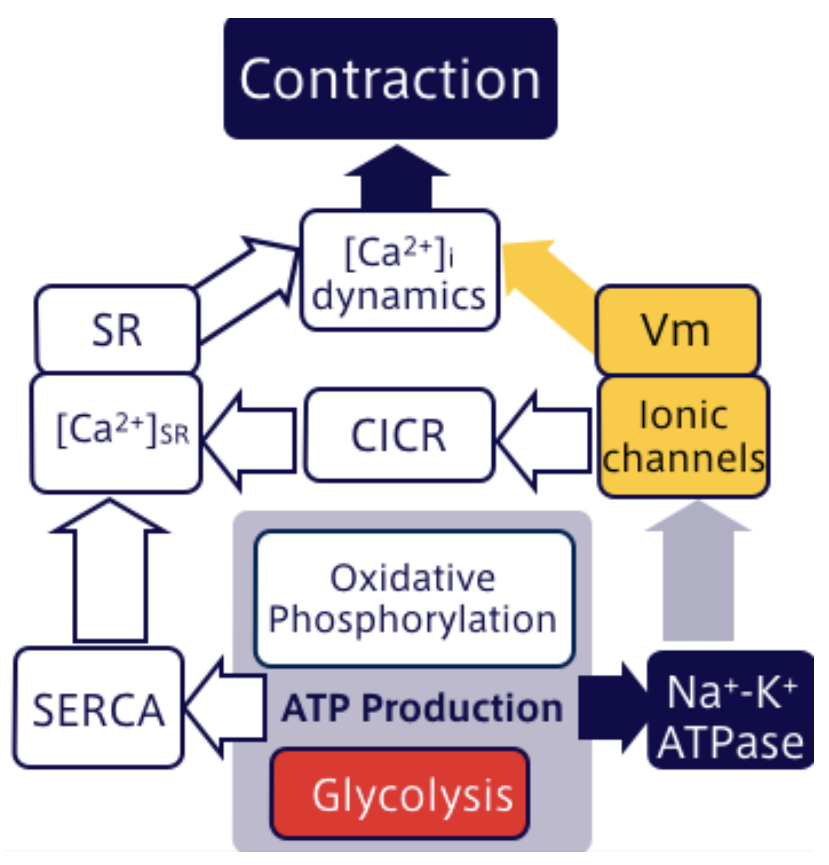

Figure 1. Schematic diagram of excitation-contraction coupling. $\mathrm{Ca}^{2+}$-induced $\mathrm{Ca}^{2+}$ release (CICR); sarcoplasmic reticulum (SR); boltage of membrane (Vm); SR Ca ${ }^{2+}$ ATPase (SERCA); intracellular $\mathrm{Ca}^{2+}$ concentration $\left(\left[\mathrm{Ca}^{2+}\right]_{\mathrm{i}}\right) ; \mathrm{Ca}^{2+}$ concentration in SR $\left(\left[\mathrm{Ca}^{2+}\right]_{\mathrm{SR}}\right)$.

of contractility under anoxic conditions. We integrated various characteristics of energy metabolism in guinea pig fetal ventricular cells based on fetal and adult ventricular cell models. The integrated model reflects dynamic changes in the ventricular cells under anoxic conditions, with both the intracellular ATP concentration and contraction maintained under anoxic conditions for a longer period in the fetal model than in the adult model. On the basis of our simulation, we predict that increased hexokinase activity and decreased total creatine content contribute to prolonged maintenance of sarcomere 
contraction in the models with smaller fractions of mitochondria.

\section{Methods}

We previously represented quantitative changes in various ionic components as the densities of the components through developmental stages relative to those in the adult stage [1,2]. Briefly, our simulations demonstrated that the spontaneous action potential (AP) in early embryonic development disappeared during the later stages, and we integrated the developmental changes in the relative current densities into the Kyoto model to represent changes in the AP [3].

Table 1. Model components modified to represent fetal guinea pig ventricular cells

\begin{tabular}{lll}
\hline & Fetus & Adult \\
\hline$I_{\mathrm{CaL}}$ & 0.78 & 1.0 \\
$I_{\mathrm{Kr}}$ & 0.30 & 1.0 \\
$I_{\mathrm{Ks}}$ & 0.30 & 1.0 \\
$\mathrm{Na}^{+} / \mathrm{Ca}^{2+}$ exchange & 1.0 & 1.0 \\
$\mathrm{SR} \mathrm{Ca}^{2+}$ pump & 0.7 & 1.0 \\
$\mathrm{RyR} \mathrm{channel}_{\mathrm{SR} \mathrm{Ca}}^{2+}$ transfer & 0.7 & 1.0 \\
$\mathrm{SR} \mathrm{Ca}{ }^{2+}$ leak & 0.7 & 1.0 \\
$\mathrm{CICR}$ factor & 0.7 & 1.0 \\
\hline
\end{tabular}

In the present study, we defined "fetus" as 44-65-dayold fetal guinea pigs because the relative current densities were obtained from fetal guinea pigs at 44-55 days after conception [4], and concentrations of glycolytic intermediates were obtained from 60-65-day-old fetal guinea pig ventricular cells [5]. The parameters listed in Table 1 were modified to represent ventricular cells in the fetal guinea pig heart.

Recently, dynamic models of the glycolytic pathways $[6,7]$ were incorporated into the Kyoto model to reproduce a hypoxic reaction in a myocardial cell [8]. Briefly, the total concentration of cytoplasmic NAD and NADH was set to $20 \%$ of the mitochondrial NAD and $\mathrm{NADH}$ concentrations, and rate constants for creatine kinase (CK), $k_{\mathrm{f}}$ and $k_{\mathrm{b}}$, were set to $8.025 \times 10^{-2}\left(\mathrm{mM}^{-2} \mathrm{~ms}^{-}\right.$ $\left.{ }^{1}\right)$ and $4.835 \times 10^{-8}\left(\mathrm{mM}^{-1} \mathrm{~ms}^{-1}\right)$, respectively [8]. In addition, the activities of all glycolytic enzymes were multiplied by 0.2 to reflect the ratio of glycolysis and oxidative phosphorylation in ventricular cells. The initial concentrations of glycolytic intermediates were set to the concentrations observed in 60-65-day-old fetal guinea pig ventricles [5]. In addition, the relative activity of ATP production by oxidative phosphorylation was decreased to 2.5 , as we decreased the fraction of mitochondrial volume to cellular volume by half in the adult model.
The fetal and adult models without the glycolytic pathway, which are defined as the "original" models, were first simulated for $600 \mathrm{~s}$ with no external stimulus. We then applied an external stimulation of $-38 \mathrm{pA} / \mathrm{pF}$, which is $-8000 \mathrm{pA}$ divided by the cell capacitance of the original Kyoto model (211.2 pF), at a frequency of $1.0 \mathrm{~Hz}$ for $600 \mathrm{~s}$ to pace the model. The traces for membrane potentials, intracellular $\mathrm{Ca}^{2+}$ concentrations, and sarcomere lengths were obtained after pacing the models. The total ATP consumed per beat by myosin-ATPase, SR-Ca ${ }^{2+}$ ATPase, and $\mathrm{Na}^{+}-\mathrm{K}^{+}$ATPase was computed by integrating the ATP consumption rates from 0 to $500 \mathrm{~ms}$.

The fetal and adult models with the glycolytic pathway, which are defined as the "modified" models, were first simulated for $12 \mathrm{~h}$ to allow the concentrations of all glycolytic intermediates to reach a quasi-steady state. The intracellular concentrations of all glycolytic intermediates, creatine, and phosphocreatine (PCr); the cytosolic concentration of AMP; and the cytosolic and mitochondrial concentrations of NAD, NADH, ATP, $\mathrm{ADP}$, and inorganic phosphate $(\mathrm{Pi})$ were extracted and integrated into the model. The model was simulated for $600 \mathrm{~s}$ with no external stimuli, and then paced with an external stimulation of $-38 \mathrm{pA} / \mathrm{pF}$. After pacing, the oxygen concentration was set to $0 \mathrm{mM}$ to simulate anoxic conditions. The simulated traces for ATP and PCr concentrations, changes in action potential duration (APD), and amplitudes of sarcomere shortening were obtained.

The glycogen concentration, relative activity of hexokinase, and total concentrations of creatine and $\mathrm{PCr}$ were varied to determine their contributions to the maintenance of ATP concentrations under anoxic conditions in both fetal and adult ventricular cells. The concentration of glycogen was increased 5-fold over the control (21 mM) [9], the relative activity of hexokinase was varied from $50 \%$ to $300 \%$ of the control [10], and the total concentration of creatine was decreased to $50 \%$ of the control [5]; all of these characteristics were previously reported as specific characteristics of fetal guinea pig ventricular cells. We plotted the time at which the amplitude of sarcomere shortening decreased to below $5 \%$ of the amplitude under normoxic conditions against the changes in glycogen concentration, relative activity of hexokinase, and total concentration of creatine to demonstrate the contribution of the changes in parameters to maintaining contractility under anoxic conditions. In addition, we increased the fraction of the mitochondrial volume from $11.5 \%$ to $23 \%$ by increments of $1.15 \%$ and varied the relative activity of hexokinase and total concentration of creatine to further demonstrate their contributions to the fetal model with different mitochondrial volumes.

All simulations were based on the Dormand-Prince method as implemented in E-Cell Simulation Environment version 3 [11]. 


\section{Results and discussion}

The fetal ventricular cell model exhibited shorter amplitudes of both $\mathrm{Ca}^{2+}$ transients and sarcomere length (Figure 2). The relative activities of both $I_{\mathrm{Kr}}$ and $I_{\mathrm{Ks}}$ in the fetal model were set to $30 \%$ of those in the adult model, which resulted in a longer APD for the fetal ventricular cell model $(225 \mathrm{~ms})$ than for the adult ventricular cell model (176 ms). Similarly, setting the relative activity of $I_{\mathrm{CaL}}$ to 0.76 and all SR-related ionic components to 0.7 of the adult model resulted in shorter $\mathrm{Ca}^{2+}$ transients $(63 \%$ of adult) and sarcomere length ( $70 \%$ of adult) in the fetal ventricular cell model.
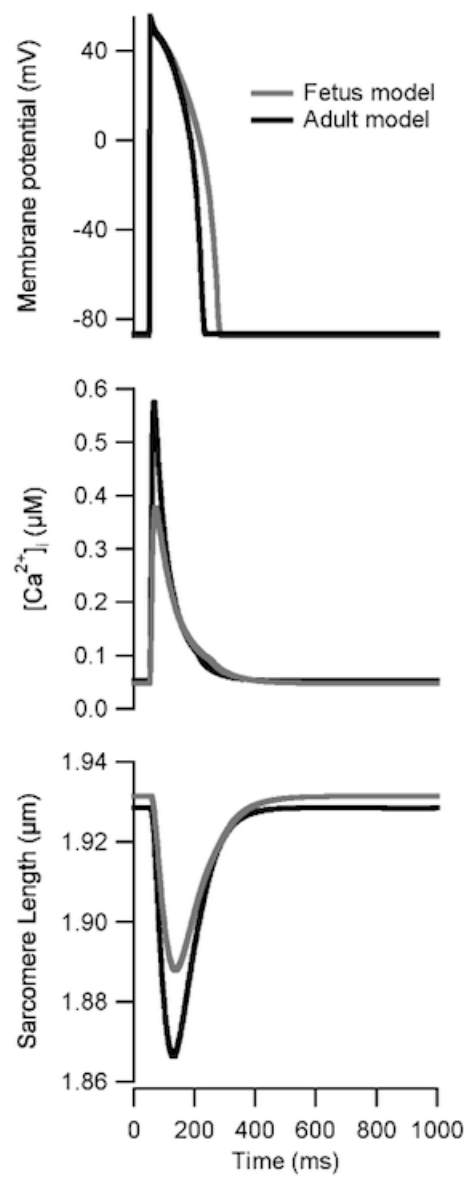

Figure 2. Simulated action potentials $(\mathrm{mV}), \mathrm{Ca}^{2+}$ transients, and sarcomere lengths of fetal and adult ventricular cells. Light and dark lines represent traces simulated with fetal and adult models, respectively.

Various characteristics specific to fetal ventricular cells contributed to prolonged maintenance of contraction (Figure 3). In Figure 3, the $y$-axis represents the time when amplitude of sarcomere shortening fell to below 5\% of the amplitude under normoxic conditions ( $\left.\mathrm{t}_{0.05}, \mathrm{~min}\right)$.
The simulation was conducted with both the fetal and adult models representing the anoxic environment with (A) increased glycogen (GLY) content up to 5-fold, (B) increased relative hexokinase (HK) activity up to 3-fold, and (C) decreased total concentrations of creatine and $\mathrm{PCr}$ ([CR]) to $50 \%$.
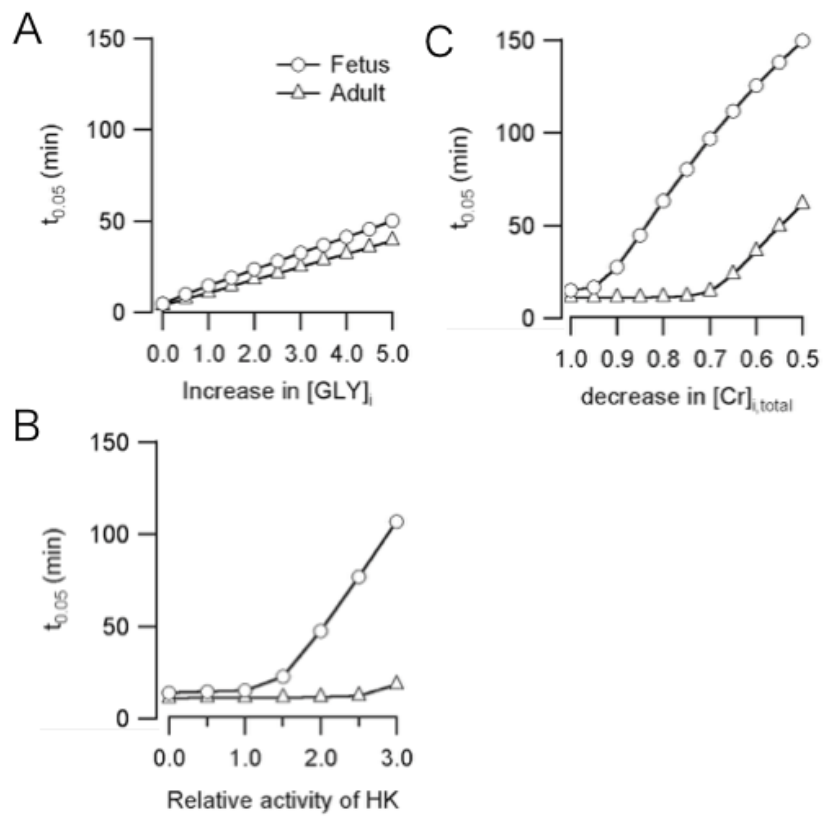

Figure 3. Contribution of various factors characteristic of fetal ventricular cells to the maintenance of contraction under anoxic conditions.

Increasing the glycogen concentration 5-fold prolonged cellular contraction to $50 \mathrm{~min}$ in the fetal model and approximately $39 \mathrm{~min}$ in the adult model (Figure 3A). Similarly, increasing the relative hexokinase activity by 3 -fold contributed to maintenance of contraction for $108 \mathrm{~min}$ in the fetal model and $18 \mathrm{~min}$ in the adult model (Figure 3B), and decreasing the total concentrations of creatine and $\mathrm{PCr}$ maintained contraction for $150 \mathrm{~min}$ in the fetal model and $62 \mathrm{~min}$ in the adult model (Figure 3C).

\section{Conclusions}

We constructed a model of energy metabolism in fetal ventricular cells. Characteristic fetal factors prolonged the time that contraction was maintained.

\section{Acknowledgements}

This work was supported by funds from the Yamagata Prefectural Government and Tsuruoka City and Keio Gijuku Academic Development Funds. 


\section{References}

[1] Itoh H, Naito Y, Tomita M. Simulation of developmental changes in action potentials with ventricular cell models. Syst Synth Biol 2007;1:11-23.

[2] Okubo C, Sano HI, Naito Y, Tomita M. Contribution of quantitative changes in individual ionic current systems to the embryonic development of ventricular myocytes: a simulation study. J Physiol Sci 2013;63:355-67.

[3] Matsuoka S, Sarai N, Kuratomi S, Ono K, Noma A. Role of individual ionic current systems in ventricular cells hypothesized by a model study. Jpn J Physiol 2003;53:105-23.

[4] Kato Y, Masumiya H, Agata N, Tanaka H, Shigenobu K. Developmental changes in action potential and membrane currents in fetal, neonatal and adult guinea-pig ventricular myocytes. J Mol Cell Cardiol 1996;28:1515-22.

[5] Rolph TP, Jones CT. Regulation of glycolytic flux in the heart of the fetal guinea pig. J Dev Physiol 1983;5:31-49.

[6] Lueck JD, Fromm HJ. Kinetics, mechanism, and regulation of rat skeletal muscle hexokinase. J Biol Chem 1974;249:1341-7.

[7] Lambeth MJ, Kushmerick MJ. A computational model for glycogenolysis in skeletal muscle. Ann Biomed Eng 2002;30:808-27.

[8] Amano A, Tomita S, Matsuoka S, Shimayoshi T, Lu J, Matsuda T. Construction of myocardial cell model including glycolysis model for reproducing hypoxic reaction. IEICE Trans D 2010;J93-D:398-408.

[9] Hirakow R, Gotoh T. Quantitative studies on the ultrastructural differentiation and growth of mammalian cardiac muscle cells. II. The atria and ventricles of the guinea pig. Acta Anat (Basel) 1980;108:230-7.

[10] Barrie SE, Harris P. Myocardial enzyme activities in guinea pigs during development. Am J Physiol 1977;233:H707-10.

[11] Takahashi K, Kaizu K, Hu B, Tomita M. A multialgorithm, multi-timescale method for cell simulation. Bioinformatics 2004;20:538-46.

Address for correspondence:

Hitomi I. Sano

5322 Endo, Fujisawa

Kanagawa 252-0882, Japan

ducky@sfc.keio.ac.jp 